\title{
Cambio climático y ecosistemas estratégicos: percepciones de estudiantes universitarios
}

Farid L. Rodríguez-Pacheco ${ }^{\star}$, Dania L. Mejía-Rodríguez ${ }^{2}$, y Jorge O. Sánchez-Buitrago ${ }^{3}$

(1) Universidad del Magdalena, Instituto Nacional de Formación Técnica Profesional Humberto Velásquez García. Grupo de Investigación en Gestión Pedagógica Transformadora - GEPET., Calle 29i-5 № 21D-37, C.P. 470001.

Santa Marta, Colombia. (correo-e: frodriguez@unimagdalena.edu.co)

(2) Universidad Simón Bolívar, Departamento de Ciencias Sociales y Humanas. Dirección: Cra. 59 № 59-65,

C.P. 080002. Barranquilla, Colombia. (correo-e: dmejia22@unisimonbolivar.edu.co)

(3) Universidad del Magdalena, Grupo de Investigación en Gestión Pedagógica Transformadora - GEPET.,

Av. del Libertador No 19-98, C.P. 470002, Santa Marta, Colombia. (correo-e: joswaldosanchez@unimagdalena.edu.co)

* Autor a quien debe ser dirigida la correspondencia.

Recibido May. 23, 2021; Aceptado Jul. 20, 2021; Versión final Ago. 10, 2021, Publicado Dic. 2021

\section{Resumen}

Se presenta los resultados de una investigación cuantitativa, descriptiva - transversal sobre percepciones acerca del cambio climático entre estudiantes universitarios residentes en la Región Caribe de Colombia. Se seleccionaron 1243 sujetos mediante muestreo aleatorio estratificado $(1-\alpha=97 \%)$. Se utilizó la escala de percepciones sobre el cambio climático $(\alpha=0,94)$. Los hallazgos evidencian relación significativa pero débil entre las percepciones existentes y el lugar de residencia $\left(X^{2}:(p \leq 0,001\right.$; $V$ de Cramer $<0,2)$. Esta se ve reflejada en percepciones afines con el reconocimiento del cambio climático entre residentes en ecosistemas con mayor afectación, pero que varían en torno a precauciones, consecuencias y acciones de mitigación, teniendo en cuenta procesos educativos, socioeconómicos y gubernamentales desarrollados en cada territorio. Los resultados visibilizan la importancia de nuevas posibilidades investigativas sobre factores que median interacciones individuo-comunidad-entorno y su papel en las percepciones y acciones de los universitarios frente al cambio climático.

Palabras clave: cambio climático; ecosistemas estratégicos; percepciones; educación superior; estudiantes universitarios

\section{Climate change and strategic ecosystems: perceptions of university students}

\begin{abstract}
Quantitative research was developed, with a transversal, descriptive design and the results are presented in this paper. The aim of study was to analyze climate change perceptions among university students from the Caribbean region of Colombia. A sample of 1243 students were selected through stratified random sampling $(1-\alpha=97 \%)$. Climate change perceptions scale was used $(\alpha=0,94)$. Main findings indicate a significant but weak relationship between university students' perceptions and their residence place $\left(X^{2}: p \leq 0,001\right.$; Cramer's $\mathrm{V}<0,2)$. However, these perceptions differ about precautions, consequences, mitigation and adaptation actions. They could be related to educational, socioeconomical and governmental processes developed in each territory. Finally, new possibilities of research are identified. Those could be directed to analyze mediators of individual-communities-environment interactions and climate change perceptions between university students.
\end{abstract}

Keywords: climate change; strategic ecosystems; perceptions; higher education; university students 


\section{INTRODUCCIÓN}

Las altas emisiones de gases de efecto invernadero (GEI), la deforestación y sobreexplotación de recursos naturales han exacerbado las condiciones climáticas de la tierra (Buotte et al., 2016). En consecuencia, se ha producido el cambio climático, alterando la composición atmosférica e incrementado la temperatura media del planeta. Entre sus efectos están el descongelamiento de los glaciares, aumento del nivel del mar, prolongación de los periodos de sequías, inundaciones y afectaciones en la salud humana (Amoo y Fagbenle, 2020). Asimismo, impacta sobre la seguridad y soberanía alimentaria e influye en la elevación de los niveles de pobreza y migración climática.

Para Bárcena et al. (2020) el cambio climático se manifiesta con una relación asimétrica fundamental entre emisiones y vulnerabilidad, es decir, los países de menor ingreso son los que menos emiten GEI, sin embargo, están y seguirán recibiendo en mayor medida las consecuencias, en ocasiones porque no cuentan con los recursos institucionales o financieros para adaptarse frente a la variación de la temperatura. Coherente con ello, el Caribe colombiano, al ser una región caracterizada por desarrollar actividades agrícolas, ganaderas y turísticas, se presenta como una región altamente vulnerable ante los efectos del cambio climático. Su territorio ha presentado aumento del nivel del mar (3-5 mm/año), inundaciones, sequías, erosión, sedimentación, pérdida de la biodiversidad y derretimiento de los picos nevados de la Sierra Nevada de Santa Marta. El panorama se complejiza por las presiones antropogénicas sobre la infraestructura natural y la capacidad de provisión de servicios ecosistémicos (Hernández-Narváez et al., 2019).

Los ecosistemas son complejos dinámicos conformados por plantas, animales, micro-organismos y seres inertes que se relacionan entre sí como una unidad funcional. La importancia de los ecosistemas radica en sus servicios de aprovisionamiento; regulación y mantenimiento, y servicios culturales para la humanidad. Dentro de los servicios de aprovisionamiento se destacan, entre otros, los bienes tangibles como los alimentos, el suministro de agua dulce, la madera y fibras para el consumo humano. Por su parte, los servicios de regulación y mantenimiento contribuyen al mejoramiento de la calidad del aire, la purificación de las fuentes hídricas, regulación del clima, control de la erosión, la polinización, fertilización del suelo y control de plagas. De otro lado, los servicios culturales brindan identidad cultural a las comunidades derivadas de su relación con la naturaleza, por ejemplo: la espiritualidad, inspiración artística e intelectual, la recreación y turismo, la vocación a actividades relacionadas con los ecosistemas (pesca, caza, siembra) y los estilos de vida (LunaCabrera, et al., 2020; Asmus et al., 2019).

El carácter estratégico de algunos ecosistemas radica en la prioridad de la gestión ambiental en su cuidado, protección y conservación estricta por medio de las políticas gubernamentales en razón a los servicios que prestan estos ecosistemas al sostenimiento de los procesos naturales, sociales, económicos y culturales. En este orden, los ecosistemas estratégicos deben ser entendidos como estructuras ecológicas diferenciables en el territorio donde se concentran funciones vitales en las relaciones socioecológicas significativas para la sostenibilidad de la humanidad y el planeta.

Teniendo en cuenta lo anterior, es posible plantear que el Caribe colombiano es una región de contrastantes ecosistemas naturales donde se pueden diferenciar 24 grandes divisiones ecosistémicas que incluyen manglares, ciénagas, bosques inundables, sabanas, bosques secos tropicales, formaciones desérticas y presencia de sierras, grandes ríos como el Magdalena, el Sinú y el Canal del Dique, extensas planicies litorales, fluviales y lacustres. Además, cuenta con áreas de serranías y montañas, donde se destacan la Sierra Nevada de Santa Marta, las serranías de la Macuira, de Cosinas, de San Jacinto, del Sinú, de San Lucas, de los Motilones, de San Jerónimo Ayapel y Abibes, entre otras (Rangel-Ch, 2015).

Aun con esta biodiversidad, el cambio climático es un factor determinante que amenaza con deteriorar los ecosistemas estratégicos de esta región. Se prevé la afectación de las interacciones de las especies, los ciclos de nutrientes, estructura y distribución de los ecosistemas, y la consecuente alteración en los flujos y calidad en la oferta de servicios ecosistémicos. Así, debido a la incertidumbre causada por las posibles consecuencias del cambio climático en la biodiversidad, se hace necesario alcanzar una mayor comprensión sobre las dinámicas, estructura y funcionamiento de los ecosistemas en un entorno climático cambiante.

En este punto es pertinente tener en cuenta que las características de cada ecosistema estratégico permiten la configuración de factores culturales, sociopolíticos, económicos y ambientales que confluyen en el desarrollo de las dinámicas que tienen lugar en cada uno de ellos. Aquí la acción humana no es la excepción, teniendo en cuenta que según las características del ecosistema en el que las comunidades se desarrollan e interactúan, se van consolidando pautas de comportamiento, sistemas de creencias, percepciones, y estrategias de relacionamiento con el entorno (Lemée et al., 2020; De La Rosa-Velázquez y Ruiz-Luna, 2019). De allí que, para generar acciones pertinentes para abordar el cambio climático resulta relevante comprender las particularidades del cambio climático en sus contextos sociales. 


\section{OTROS ANTECEDENTES}

Indagar sobre las percepciones acerca del cambio climático en un territorio, contribuye a la comprensión contextualizada en torno al grado de sensibilidad, información y abordaje de este fenómeno (Cruz y Páramo, 2020; Lambrechts y Ceulemans, 2013). Las percepciones configuran la comprensión y las explicaciones sobre la realidad, según los conocimientos y actitudes construidos desde la interacción individuo-entorno (Yu et al., 2013). Estas ofrecen insumos para documentar los procesos de degradación ecológica y la forma en que las sociedades los interpretan a través de los valores, la cultura, creencias y relación sociedad-naturaleza. Asimismo, se evidencia cómo las poblaciones experimentan y se adaptan a los cambios socioecológicos de los ecosistemas (Viguera et al., 2019; Forero et al., 2014).

Al respecto, es posible anotar que las investigaciones sobre percepciones acerca del cambio climático han venido aumentando, toda vez que los saberes de las comunidades con respecto a estos factores contribuyen a complementar la investigación científica, como también al diseño de medidas de mitigación y adaptación al clima cambiante (Ventura et al., 2020; Hansen, 2012). Esto, basados en que cuando un problema no es socialmente percibido y asumido, termina siendo irrelevante en su contexto social, debido a que las comunidades no le asignan prioridad de atención (Viguera et al., 2019; Moyano et al., 2015). De igual manera, las percepciones sobre el cambio climático se configuran desde la confluencia de los significados que construyen los individuos y las dinámicas socioeconómicas, culturales, educativas e incluso gubernamentales en las que estos se ven inmersos (Schneiderbauer et al., 2021; Forero et al., 2014).

Así, una investigación sobre el cambio climático en los ecosistemas estratégicos del Caribe colombiano, es pertinente porque, este territorio es considerado como de alta vulnerabilidad ante los efectos del cambio climático, pues, según Hernández- Narváez et al., (2019), en esta región se han presentado variaciones de temperatura, cambios en los patrones de precipitaciones, y cambios en los sistemas costeros que pueden repercutir en la seguridad alimentaria, el desarrollo socioeconómico, las dinámicas de los asentamientos humanos, la infraestructura y en general en las condiciones de vida de sus habitantes. En esta línea, analizar las percepciones de las personas frente al cambio climático según los ecosistemas estratégicos en los que se desenvuelven, contribuye a fortalecer entramados teóricos y marcos de actuación que aporten a su mitigación y adaptación, basados en las características y dinámicas relacionales entre individuos, comunidades y entorno (Hernández-Diaz et al., 2021; Hansen et al., 2012).

Bajo este panorama, la universidad cobra relevancia en la comprensión de las percepciones sobre el cambio climático en los distintos ecosistemas estratégicos; dado que, sus ejes misionales trascienden la exclusiva cualificación disciplinar, orientándose también, al desarrollo de procesos pertinentes para el mejoramiento de su entorno desde distintos ámbitos, entre ellos el ambiental. Además, es un escenario propicio para la formación, sensibilización y el desarrollo de estrategias de mitigación y adaptación desde la transdisciplinariedad necesaria para abordar este fenómeno (Hess y Maki, 2019).

En este sentido, se asume a los estudiantes universitarios como participantes del estudio por su cercanía y afinidad con la cultura científica (Hernández- Diaz, et al, 2021; Yáñez, Díaz y Pérez, 2018) y por su rol como ciudadanos que viven actualmente las manifestaciones del cambio climático (Leal Filho et al., 2019). Estos actores, potencialmente pueden aportar, desde sus disciplinas, a la delimitación de estrategias efectivas frente al cambio climático (Moyano et al., 2015). Por ello, esta investigación se orienta a analizar las percepciones de los estudiantes universitarios del Caribe colombiano acerca del cambio climático, según los ecosistemas estratégicos presentes en su departamento de residencia. Esto, como una aproximación contextualizada al fenómeno, que aporte al fortalecimiento de procesos para su abordaje desde el ámbito universitario.

\section{METODOLOGÍA}

En la sección de metodología se presenta el diseño de la investigación, la población participante, el instrumento de recolección de información y el procedimiento para el procesamiento y el análisis de los datos.

Diseño: Esta investigación es de tipo cuantitativa dirigida a analizar las percepciones sobre el cambio climático entre universitarios residentes en los siete departamentos de la Región Caribe colombiana. Permite una aproximación a cómo aporta la interacción con ecosistemas estratégicos ubicados en estos departamentos sobre la percepción de los universitarios. Su alcance es descriptivo, comparativo y de corte transversal (Sánchez, 2019).

Participantes: La población está conformada por 110.136 estudiantes vinculados a universidades públicas del Caribe colombiano (MEN, 2017). La población fue organizada en siete grupos distribuidos proporcionalmente; uno por cada universidad pública, delimitando una muestra de 1243 sujetos (1- $\alpha$ : $97 \%$, error muestral: $3 \%$ ). El $52 \%$ de los participantes fueron hombres y el $48 \%$ mujeres (media de edad=20,53; Mín: 18 - Máx: 30), 
vinculados a programas de ingeniería, ciencias económicas, empresariales y ambientales, y en menor proporción a ciencias de la educación, ciencias básicas, ciencias sociales y jurídicas, y ciencias de la salud. Los participantes tenían mínimo 5 años de residencia en el departamento que registraron al momento de la medición: $20 \%$ en el departamento del Atlántico, $16 \%$ en los departamentos de Cesar y Bolívar, $15 \%$ tanto en Magdalena como en Córdoba, $13 \%$ en La Guajira y $5 \%$ en Sucre.

Instrumento: Se realizó y utilizó una adaptación colombiana del cuestionario Climate change perceptions scale (Bakaç, 2018) - Alpha de Cronbach: 0,94. Este cuenta con 22 ítems, respuestas tipo Likert, distribuidos en seis subescalas: 1. Razones del cambio climático: Reconocimiento de situaciones relacionadas con la ocurrencia del fenómeno como variaciones en la temperatura, descongelamiento de los picos nevados, sequías, degradación de la tierra y pérdida de la biodiversidad. 2. Conciencia frente al fenómeno: Percepciones sobre el estado actual del cambio climático y sus consecuencias. Indaga sobre la frecuencia y la severidad de eventos relacionados con el fenómeno y sus afectaciones en los ecosistemas. 3. Futuros escenarios: Condiciones sociales, económicas y ambientales, potencialmente derivados de la ocurrencia del cambio climático y de la falta de acciones para su mitigación y la adaptación. 4. Impacto ambiental: Percepciones sobre las consecuencias individuales y sociales derivadas del cambio climático. Así como la percepción de la sensibilidad que tienen los otros sobre la ocurrencia de este fenómeno. 5. Consecuencias: Acciones humanas que asumen los participantes a partir de la ocurrencia de este fenómeno en su territorio, así como factores que han potenciado la ocurrencia y magnitud del cambio climático. 6. Precauciones: Percepciones sobre la existencia o implementación cercana de acciones humanas dirigidas a la mitigación y la adaptación del cambio climático. Cada una se evalúa en función de tres niveles: Bajo, medio y alto. Estos se determinan a partir de la ubicación del valor total de cada subescala en centiles: Nivel bajo $\leq 25$; Nivel medio $>25$ y $\leq 75$; Nivel alto $>75$.

Procedimiento: La investigación implicó adaptar el instrumento de medición al contexto colombiano mediante traducción directa e inversa validadas por expertos, y validación de contenido por pares. Asimismo, se identificaron sus propiedades psicométricas en una aplicación de 254 sujetos. La selección de la muestra se realizó mediante un muestreo aleatorio estratificado de fijación proporcional con las universidades participantes. La caracterización sociodemográfica y la aplicación del instrumento se realizó presencialmente, durante el año 2019 (30-40 estudiantes por grupo de aplicación). En estas sesiones se realizó socialización de consentimiento informado, se brindaron indicaciones y acompañamiento para el diligenciamiento del instrumento. Posteriormente, se utilizó el software SPSS - Versión 24.0 para el procesamiento y análisis de datos. Se establecieron tendencias porcentuales de respuesta por cada subescala evaluada y se identificaron contingencias entre las percepciones de los participantes y su departamento de residencia. Asimismo, se realizó la prueba Chi cuadrado para establecer posibles relaciones entre las variables evaluadas y se halló el coeficiente $\mathrm{V}$ de Cramer para identificar su grado de asociación.

\section{RESULTADOS}

Se analizaron contingencias entre las percepciones de los universitarios sobre el cambio climático y el departamento de residencia. Estas permitieron evidenciar un mayor reconocimiento del cambio climático entre los participantes residentes en el departamento de Sucre. Esto, basado en mayores porcentajes de niveles medio y alto con respecto a las respuestas de los residentes en los demás departamentos. Además, se identifica un alto porcentaje de estudiantes de Sucre que reconocen consecuencias en las dinámicas individuales y sociales derivadas del cambio climático, así como acciones requeridas para evitar más afectaciones en el entorno derivadas de su ocurrencia (ver tabla 1).

Tabla 1: Percepciones sobre el cambio climático entre universitarios, según el departamento de residencia

\begin{tabular}{|l|l|c|c|c|c|c|c|c|}
\hline \multirow{3}{*}{ Subescala } & \multirow{2}{*}{ Nivel } & \multicolumn{9}{|c|}{ Porcentaje por departamento } \\
\cline { 3 - 10 } & & Córdoba & Atlántico & Magdalena & Sucre & Bolívar & La Guajira & Cesar \\
\hline \multirow{3}{*}{ Razones } & Bajo & 20,2 & 26,7 & 24,7 & 16,4 & 36,8 & 32,1 & 23,0 \\
\cline { 2 - 11 } & Medio & 47,5 & 47,8 & 42,9 & 54,1 & 39,8 & 44,4 & 44,0 \\
\cline { 2 - 11 } & Alto & 32,2 & 25,5 & 32,4 & 29,5 & 23,4 & 23,5 & 33,0 \\
\hline \multirow{3}{*}{ Conciencia } & Bajo & 19,9 & 24,2 & 14,4 & 9,4 & 32,3 & 23,5 & 20,6 \\
\cline { 2 - 11 } & Medio & 48,6 & 56,7 & 63,0 & 79,7 & 48,5 & 63,0 & 59,9 \\
\cline { 2 - 11 } & Alto & 31,5 & 19,1 & 22,5 & 10,9 & 19,1 & 13,6 & 19,5 \\
\hline
\end{tabular}


Tabla 1: continuación

\begin{tabular}{|c|c|c|c|c|c|c|c|c|}
\hline \multirow{2}{*}{ Subescala } & \multirow{2}{*}{ Nivel } & \multicolumn{7}{|c|}{ Porcentaje por departamento } \\
\hline & & Córdoba & Atlántico & Magdalena & Sucre & Bolívar & La Guajira & Cesar \\
\hline \multirow{3}{*}{ Precauciones } & Bajo & 21,9 & 33,9 & 38,5 & 5,0 & 35,6 & 38,9 & 38,4 \\
\hline & Medio & 54,6 & 42,7 & 39,6 & 61,7 & 37,1 & 32,7 & 37,9 \\
\hline & Alto & 23,5 & 23,4 & 22,0 & 33,3 & 27,2 & 28,4 & 23,6 \\
\hline \multirow{3}{*}{ Escenarios Futuros } & Bajo & 18,0 & 27,5 & 17,6 & 11,5 & 34,2 & 32,7 & 22,9 \\
\hline & Medio & 47,5 & 42,5 & 46,2 & 47,5 & 39,1 & 42,6 & 44,3 \\
\hline & Alto & 34,4 & 30,0 & 36,3 & 41,0 & 26,7 & 24,7 & 32,8 \\
\hline \multirow{3}{*}{ Impacto Ambiental } & Bajo & 21,4 & 19,4 & 19,2 & 8,2 & 38,1 & 30,9 & 21,4 \\
\hline & Medio & 56,0 & 54,8 & 50,5 & 59,0 & 43,1 & 49,4 & 54,2 \\
\hline & Alto & 22,5 & 25,8 & 30,2 & 32,8 & 18,8 & 19,8 & 24,4 \\
\hline \multirow{3}{*}{ Consecuencias } & Bajo & 24,7 & 34,8 & 31,9 & 14,8 & 42,6 & 42,6 & 30,5 \\
\hline & Medio & 41,2 & 39,3 & 41,2 & 62,3 & 36,1 & 38,9 & 37,4 \\
\hline & Alto & 34,1 & 25,9 & 26,9 & 23,0 & 21,3 & 18,5 & 32,0 \\
\hline
\end{tabular}

Igualmente, se resalta una tendencia hacia niveles medios de reconocimiento del cambio climático entre los participantes de los demás departamentos (subescala razones). Sin embargo, los estudiantes de Bolívar registran resultados ligeramente menores a los demás; identificándose el mayor porcentaje de participantes ubicados en niveles bajos (36,8 \%) y el menor porcentaje en niveles altos $(23,4 \%)$. Igualmente, se identifica el departamento de La Guajira con un 32,1\% ubicados en nivel bajo, $44,4 \%$ en nivel medio y $23,5 \%$ en niveles altos. En la subescala de conciencia, los estudiantes de Bolívar registran 32,3\% en nivel bajo, sugiriendo un menor reconocimiento del cambio climático como fenómeno ocurrente en su contexto. Asimismo, se observa una menor identificación de las afectaciones, los impactos, las condiciones futuras que puedan derivarse de su ocurrencia, y de las acciones pertinentes para su mitigación y adaptación. Esto se evidencia en las subescalas futuros escenarios, impacto ambiental y consecuencias, donde se presentan los mayores porcentajes en el nivel bajo $(34,2 \%, 38,1 \%$ y $42,6 \%$, respectivamente).

El 30,9\% de los estudiantes de La Guajira, registran niveles bajos de percepciones afines con la identificación de impactos del cambio climático, de igual manera en un $34,2 \%$ sobre condiciones futuras y $42,6 \%$ en la subescala consecuencias. Esto sugiere que, si se asume que el cambio climático no genera impactos en el individuo y su sociedad, es posible que esta percepción dificulte el reconocimiento de acciones humanas asociadas a su ocurrencia y de escenarios futuros relacionados con este fenómeno. En el caso de Córdoba, la subescala de conciencia presenta un $19,9 \%$ en el nivel bajo y se ubica como el departamento con el mayor porcentaje de participantes en un nivel alto de reconocimiento de las acciones humanas que han potenciado el cambio climático. Asimismo, se registran altos porcentajes frente al reconocimiento de acciones para la mitigación y adaptación del fenómeno $(34,1 \%$ en nivel alto y $41,2 \%$ en nivel medio en la subescala consecuencias).

Finalmente, se resaltan resultados de estudiantes residentes en el Atlántico, Cesar y Magdalena frente a la subescala de precauciones $(33,9 \%, 38,4 \%$ y $38,4 \%$ en niveles bajos, respectivamente). Esta subescala registra los mayores porcentajes de participantes en el nivel bajo, entendido como dificultades para reconocer acciones dirigidas a la mitigación y adaptación al cambio climático. Incluso, es posible que aun cuando se realicen acciones relacionadas con este objetivo, no se asocien con ello. Basados en las contingencias encontradas, se establecieron los estadísticos que permiten corroborar la relación entre las variables. Así, el chi cuadrado establecido para cada una de las subescalas con relación a los departamentos de residencia se registra 12 grados de libertad y una relación significativa en todos los casos $(p \leq 0,001)$ (ver tabla 2$)$.

Tabla 2. Chi cuadrado $\left(\mathrm{X}^{2}\right)$ y coeficiente v de Cramer

\begin{tabular}{|l|c|r|c|l|c|}
\hline \multirow{2}{*}{} & \multicolumn{3}{|c|}{$\mathrm{X}^{2}$} & \multicolumn{2}{c|}{ V de Cramer } \\
\cline { 2 - 6 } & Valor & Gl & $\begin{array}{l}\text { Sig. asintótica } \\
\text { (bilateral) }\end{array}$ & Valor & Sig. Aproximada \\
\hline Conciencia & 32,211 & 12 &, 001 &, 114 &, 001 \\
\hline Razones & 26,539 & 12 &, 000 &, 103 &, 000 \\
\hline Precauciones & 49,452 & 12 &, 000 &, 141 &, 000 \\
\hline Futuros escenarios & 33,731 & 12 &, 001 &, 117 &, 001 \\
\hline Impacto ambiental & 59,799 & 12 &, 000 &, 155 &, 000 \\
\hline Consecuencias & 37,008 & 12 &, 000 &, 122 &, 000 \\
\hline
\end{tabular}


Esto se corrobora al calcular el coeficiente de $\mathrm{V}$ de Cramer, estructurado en una escala de 0 a 1 , donde cero=nula relación entre variables y uno=fuerte relación entre variables. Los resultados de este coeficiente registran una relación débil pero existente (ver tabla 2) que puede estar dado porque el instrumento evalúa cada una de las percepciones de los estudiantes sobre el cambio climático desde un panorama general que si bien es aplicable al contexto no profundiza en particularidades de los territorios que podrían visibilizar una mayor significatividad estadística para la relación entre estas variables. Lo anterior teniendo en cuenta que entre los hallazgos obtenidos se registran diferencias entre las percepciones de los estudiantes según el lugar de residencia, por lo que resulta pertinente precisar qué elementos o factores de los ecosistemas estratégicos que integran cada uno de los departamentos pueden generar estas diferencias, o incluso si existen otros factores que no han sido contemplados y que podrían generar un análisis más preciso en torno a la relación entre las percepciones que se construyen acerca del cambio climático de acuerdo con los ecosistemas estratégicos donde interactúan los universitarios.

Basados en todos los hallazgos identificados se resaltan las percepciones de los participantes residentes en el departamento de Sucre como las más cercanas al reconocimiento del cambio climático y sus repercusiones. En este departamento se encuentran ecosistemas como las llanuras del Morrosquillo, los Montes de María y la Depresión Momposina. La subregión Sabanas, territorio originalmente de bosques secos tropicales, ha sido reemplazada por praderas artificiales para ganadería extensiva. Además, se ha generado desecación de ciénagas, sobrepesca, uso indiscriminado de agroquímicos y contaminación de fuentes hídricas por la producción aurífera. Estas prácticas han afectado la eficiencia de los servicios ecosistémicos del departamento, contribuyendo posiblemente a percepciones sobre la vulnerabilidad ante el cambio climático, así como una reducción de las capacidades de adaptación (Hernández-Narváez et al., 2019; Rangel, 2015; Guerrero et al., 2014).

El departamento de Bolívar, por su parte, cuenta con ecosistemas como el río Magdalena, los Montes de María, Ciénaga la Virgen, Serranía San Lucas, la Depresión Momposina, zonas de manglar y marino-costera. En su historia reciente, Bolívar fue afectado por la ola invernal de 2010-11 con inundaciones en la ecorregión Canal del Dique (con pérdidas estimadas superiores al $5 \%$ del Producto Interno Bruto (PIB) departamental). Además, en Cartagena, capital del departamento, se registra aumento del nivel del mar, erosión costera, mares de leva, inundaciones y veranos intensos como principales efectos del cambio climático. Sin embargo, los estudiantes encuestados de este territorio suelen registrar datos relacionados con percepciones más alejadas al reconocimiento de este fenómeno. Esto puede obedecer a que, en su mayoría, los participantes residen en la ecorregión Montes de María, que ha tenido menor afectación que la zona marino-costera y la ecorregión canal del Dique (Gobernación de Bolívar et al., 2018).

En los departamentos de La Guajira y Cesar se encuentran la Serranía de Macuira, la Sierra Nevada de Santa Marta, el valle aluvial de los ríos Cesar y Ranchería, la Serranía de Perijá, Cerro Pintao, Ciénaga de Zapatosa y el Santuario de Flora y Fauna de los Flamencos. Estos ecosistemas presentan pérdida de la biodiversidad por el desarrollo de agricultura intensiva con excesivo uso de agroquímicos y poca rotación de cultivos. No obstante, los estudiantes residentes en estos departamentos reflejan niveles bajos en la subescala razones y precauciones, posiblemente asociados a que estos territorios son considerados los principales exportadores de carbón de Suramérica. Ambos cubren el $90 \%$ de la producción nacional del carbón extraído para exportación, representando el 42,4 \% del PIB de La Guajira y el 52 \% del PIB del Cesar (Bayona, 2016). Esto puede conllevar a una menor percepción de medidas para mitigar el cambio climático, pues la producción y consumo de carbón es una de las prácticas que más emite GEl.

El departamento del Atlántico cuenta aproximadamente con 26.000 hectáreas de bosques $(7,96 \%$ de la superficie departamental) y posee cuerpos de agua como la ciénaga de Mallorquín, el canal del Dique y el embalse del Guájaro. Estos ecosistemas están siendo afectados por la deforestación y la desecación de ciénagas, intensificando los impactos del cambio climático en la región (Hernández-Narváez et al., 2019; Rangel-Ch, 2015). Sin embargo, los participantes residentes en este territorio registran niveles de percepción bajos sobre las precauciones frente al fenómeno. Esta tendencia puede asociarse a que no existen áreas de protección de carácter nacional y a las escasas acciones para la conservación de ecosistemas de humedales; así como acciones departamentales aisladas para la educación ambiental, la gestión del cambio climático y su incorporación al ordenamiento ambiental y territorial.

Según la Corporación Autónoma Regional del Magdalena - CORPAMAG (2016), el departamento cuenta con ecosistemas estratégicos declarados como reserva de la biósfera y humadales Ramsar como la Ciénaga Grande de Santa Marta, Los Valles y Colinas del Ariguaní, Humedales del Sur, zona marino-costera y la Sierra Nevada de Santa Marta. Sin embargo, Los estudiantes residentes en este territorio registran tendencias hacia niveles de percepción bajo en precauciones, posiblemente relacionadas con debilidades en la ejecución de proyectos educativos ambientales. Lo anterior, teniendo en cuenta que los procesos de educación ambiental implementados suelen enfatizar en la transmisión de conocimiento disciplinar que si bien es importante, 
resulta insuficiente para la apropiación social del conocimiento, la contextualización y transformación de las realidades ambientales.

Los estudiantes del departamento de Córdoba registran el porcentaje más alto de percepciones sobre el reconocimiento de acciones de mitigación y adaptación al cambio climático. Este comportamiento puede asociarse a la implementación de estrategias relacionadas con la gestión del riesgo, la socialización de puntos críticos por amenazas, la promoción de cultura de prevención y la difusión de información sobre causas y efectos de fenómenos ambientales en el departamento (Schneiderbauer et al., 2021). Además, se han impulsado proyectos de educación ambiental no formal para la conservación de los ecosistemas estratégicos. Entre ellos, se destacan iniciativas comunitarias de educación ambiental lideradas por la Asociación de Pescadores, Campesinos, Indígenas y Afrodescendientes (ASPROGIC) para el desarrollo comunitario y la adaptación al cambio climático de la Ciénaga Grande del Bajo Sinú. Ejemplo de ello son los sistemas agroecológicos en Diques Altos (SADAS) y los agroecosistemas biodiversos familiares (ABIF). Estos se diseñan con especies vegetales nativas combinando saberes ancestrales con ciencia y tecnología, para promover sistemas resilientes a los cambios de temperatura, inundaciones y sequías, y restauración de bosque de galería asociado a las riberas del río Sinú. La Universidad de la Costa - CUC y Corporación Autónoma Regional de los Valles del Sinú y de San Jorge - CVS (2016) destacan que se han propiciado espacios de comercialización e intercambio de bienes y servicios agroecológicos mediante una estrategia creada por ASPROCIG basada en una certificación para fortalecer lazos entre productores y consumidores, garantizando el acceso a productos agroecológicos y mejorando las ganancias de las familias campesinas.

\section{DISCUSIÓN FINAL}

Las investigaciones actuales sobre cambio climático en contextos educativos en Latinoamérica han sido abordadas mediante estudios de percepciones y representación social, aplicadas fundamentalmente a la población juvenil y evidenciando que prevalecen concepciones erróneas sobre el cambio climático y confusión de este fenómeno con otras problemáticas ambientales (Cruz y Páramo, 2020). Estas concepciones erróneas y confusiones acerca del cambio climático pueden sustentar los bajos porcentajes de reconocimiento de precauciones, causas y estrategias de mitigación del cambio climático que presentan los estudiantes residentes en los departamentos del Cesar, La Guajira y Atlántico.

El mayor reconocimiento del cambio climático entre los estudiantes universitarios se encuentra en los residentes del departamento de Sucre, donde se presentó un alto porcentaje de participantes que reconocen las consecuencias y las acciones para mitigación y adaptación del territorio. Estos resultados pueden estar dados porque los ecosistemas estratégicos del departamento, tales como: los Montes de María, la Depresión Momposina y los bosques secos tropicales, han sido modificados sustancialmente para implementar actividades económicas como la ganadería extensiva, sobrepesca y la explotación aurífera. Estos hallazgos son coherentes con los planteamientos de Viguera et al., (2019) respecto a que las percepciones sobre el cambio climático entre estudiantes son mayores en los departamentos en cuyos ecosistemas estratégicos haya modificaciones antropogénicas de su estructura y funcionamiento.

No obstante, la vulnerabilidad de los ecosistemas estratégicos y los consiguientes riesgos del cambio climático no garantiza la generación de hábitos de comportamiento de protección y conservación por parte de los habitantes que residen alrededor de los ecosistemas estratégicos en riesgo (Lemée et al., 2020). Así, aunque los ecosistemas estratégicos del Caribe colombiano y Latinoamérica presentan gran sensibilidad es sus activos naturales y según Bárcena et al., (2020), para el 2050, el cambio climático representará un costo económico entre el $1,5 \%$ y el $5 \%$ del PIB regional actual aún se registran hallazgos asociados a percepciones alejadas del reconocimiento del cambio climático y de la generación de acciones para su mitigación y adaptación.

Asimismo, como sugiere Schneiderbauer et al. (2021) las percepciones sobre el cambio climático en los estudiantes universitarios también se ven condicionadas por procesos educativos, sociales y políticas gubernamentales. Por ejemplo, en Córdoba se han desarrollado programas de promoción de la cultura de prevención y difusión de las problemáticas ambientales, la gestión del riesgo e implementación de iniciativas comunitarias de educación ambiental para la conservación de los ecosistemas estratégicos del departamento, lo cual ha incidido en los altos porcentajes de reconocimiento de acciones de mitigación y adaptación al cambio climático en los estudiantes. Por el contrario, las deficiencias en la ejecución de proyectos educativos ambientales en el departamento del Magdalena pueden repercutir en las tendencias hacia niveles de percepción bajo en precauciones del cambio climático en los universitarios. De igual forma, la no declaración de áreas de protección y la falta de incorporación de determinantes ambientales al ordenamiento territorial pueden influir en los bajos niveles de percepción sobre la escala de precaución del cambio climático que se refleja en los estudiantes del departamento del Atlántico (Forero et al., 2014).

Los postulados de Schneiderbauer et al. (2021) y Forero et al. (2014) se reafirman con los planteamientos de Hess y Maki (2019) al demostrar que los estudiantes universitarios que recibieron un curso académico con 
contenidos relacionados con el cambio climático pasaron del escepticismo o la incertidumbre a una creencia ambiental asertiva acerca del fenómeno. Por lo tanto, la investigación apoya una política educativa que contenga como requisito curricular la educación sobre el clima.

Los referentes aquí descritos sugieren la importancia de tener en cuenta que la interacción con procesos y acciones relacionadas con el cambio climático pueden repercutir en las percepciones de los participantes frente a este fenómeno, sus causas e impactos. Asimismo, promueve nuevos interrogantes de investigación, entre ellos: ¿Qué atribuciones dan los participantes a los cambios que se han generado en sus ecosistemas estratégicos? ¿Qué acciones posibilitan el acercamiento de la población con los ecosistemas estratégicos? y ¿Qué factores no han sido contemplados y podrían generar un análisis más preciso en torno a la relación entre las percepciones sobre el cambio climático y los ecosistemas estratégicos donde interactúan los individuos? De igual forma, se visibiliza la necesidad de abordar el análisis institucionalmente, respecto a las gestiones académicas de la universidad frente a la educación ambiental. Esto como insumo para potenciar la calidad de las actuaciones en la mitigación y adaptación al cambio climático en proyectos ambientales universitarios, proyectos ambientales escolares, proyectos ciudadanos de educación ambiental e iniciativas afines.

\section{CONCLUSIONES}

De acuerdo con el trabajo presentado y a los resultados obtenidos, se pueden plantear las siguientes conclusiones principales:

1.- Las percepciones sobre el cambio climático entre estudiantes residentes en territorios cuyos ecosistemas estratégicos han sido afectados en su estructura y funcionamiento ilustran tendencias hacia un mayor reconocimiento de este fenómeno y sus consecuencias. Sin embargo, las percepciones en torno a estrategias para su adaptación, su mitigación y las acciones humanas pertinentes para su atención pueden variar según las interacciones de los individuos con los procesos socioeconómicos, educativos y gubernamentales que dinamizan cada contexto. Esto coincide con investigaciones previas donde se resalta que la comprensión del fenómeno y la percepción sobre medidas asociadas a su ocurrencia son priorizadas de manera coherente con dinámicas del contexto donde interactúan los individuos. En este caso, en los territorios donde están inmersos los estudiantes universitarios.

2.- Se registran tendencias hacia un mayor reconocimiento del cambio climático como un fenómeno que requiere ser atendido de manera participativa, así como una mayor percepción de acciones para la mitigación y adaptación de este entre estudiantes universitarios residentes en territorios donde se han fortalecido acciones de socialización, sensibilización y protección ambiental tanto a nivel educativo como comunitario y gubernamental.

3.- En departamentos como Cesar, La Guajira y Atlántico, a pesar de tener registros de investigaciones previas sobre afectaciones en la biodiversidad de sus ecosistemas estratégicos, se evidencian bajos porcentajes sobre reconocimiento de precauciones, estrategias de mitigación y causas del cambio climático. En estos territorios es pertinente tener en cuenta que sus principales actividades económicas, si bien pueden afectar el equilibrio de los ecosistemas estratégicos, también representan la mayor fuente de ingreso para los departamentos. Complementariamente, aunque se encuentran avances en actividades para la protección ambiental, no se identifican registros que sustenten la consolidación de estas, ni su apropiación por parte de los integrantes de la comunidad. Estas dinámicas del contexto son susceptibles de ser analizadas para profundizar en la comprensión de las tendencias en las que coinciden los territorios mencionados.

4.- El abordaje cuantitativo de la investigación permitió una aproximación al análisis de las percepciones entre universitarios de la región Caribe colombiana sobre el cambio climático. Sin embargo, las diferencias identificadas entre las percepciones de los estudiantes de acuerdo con los territorios donde residen, y las características de los ecosistemas estratégicos presentes en ellos visibilizan la importancia de generar aproximaciones cualitativas que pueden enriquecer el abordaje de este tema desde la identificación de significados y procesos de construcción tanto individuales como comunitaria que estén configurando tales percepciones. Estos insumos pueden contribuir al fortalecimiento de referentes útiles para la configuración de acciones pertinentes en el abordaje del cambio climático desde los estudiantes y desde otros actores que interactúan en los territorios que han sido objeto de la investigación.

\section{AGRADECIMIENTOS}

Los autores agradecen el apoyo del proyecto "Conocimientos, percepciones y creencias acerca del cambio climático en estudiantes universitarios de la Región Caribe de Colombia" subvencionado por la convocatoria para la financiación de proyectos de investigación, desarrollo experimental, innovación y creación artística y cultural - FONCIENCIAS 2017 de la Universidad del Magdalena, Colombia. 


\section{REFERENCIAS}

Amoo, L. M., y Fagbenle, R. L., Climate change in developing nations of the world, In Applications of Heat, Mass and Fluid Boundary Layers, 437-471, Woodhead Publishing, Cambrigde, United Kingdom (2020).

Asmus, M. L., Nicolodi, J., Anello, L. S., y Gianuca, K., The risk to lose ecosystem services due to climate change: A south American Case, https://doi.org/10.1016/j.ecoleng.2017.12.030, Ecological Engineering, 130, 233-241 (2019).

Bakaç, E., Engineering Faculty Students' Perceptions on Climate Change 10.13189/eer.2018.060404, Environment and Ecology Research, 6(4), 240-247 (2018).

Bárcena, A., y otros tres autores., La emergencia del cambio climático en América Latina y el Caribe: ¿Seguimos esperando la catástrofe o pasamos a la acción?, CEPAL, (2020)

Bayona, E. M., Producción de carbón y crecimiento económico en la región minera del Caribe colombiano, http://dx.doi.org/10.14482/ecoca.17.8452, Revista de Economía del Caribe, 17, 1-38 (2016).

Buotte, P.C., Peterson, D. L., McKelvey, K. S., y Hicke, J. A., Capturing subregional variability in regional-scale climate change vulnerability assessments of natural resources, http://dx.doi.org/10.1016/j.jenvman.2015.12.017, Journal of Environmental Management, 169, 313-318 (2016).

CORPAMAG-Corporación Autónoma Regional del Magdalena, Plan de Acción Institucional 2016-2019, 1-147 (2016).

CUC-Universidad de la Costa y CVS-Corporación Autónoma Regional de los Valles del Sinú y de San Jorge, Plan Departamental de Adaptación al Cambio Climático para el Departamento de Córdoba 2016-2027, 1-74 (2016).

Cruz, N., y Páramo, P., Educación para la mitigación y adaptación al cambio climático en América Latina, https://doi.org/10.5294/edu.2020.23.3.6, Educación y Educadores, 23(3), 469-489 (2020).

De La Rosa-Velázquez., M. I., y Ruiz-Luna, A., Valoración social de los servicios ecosistémicos de humedales costeros: Estado actual y perspectivas, http://dx.doi.org/10.15446/abc.v25n3.80387, Acta Biológica Colombiana, 25(3), 403-413 (2019).

Forero, E. L., Hernández, Y. T., y Zafra, C. A., Percepción latinoamericana de cambio climático: Metodologías, herramientas y estrategias de adaptación en comunidades locales: Una revisión, https://doi.org/10.31910/rudca.v17.n1.2014.942, Revista U.D.C.A Actualidad \& divulgación científica, 17(1), 73-85 (2014).

Guerrero, R., Flórez, J., y García, L. Estado y prospectiva de las posibilidades de mitigación de impacto del cambio climático en la región Costa Atlántica, 19 (1), 121-146, Bogotá, Colombia, (2014).

Gobernación de Bolívar, UNGRD-Unidad Nacional para la Gestión del Riesgo de Desastres y PNUD-Programa de las Naciones Unidas para el Desarrollo Colombia., Plan departamental de gestión del riesgo de Bolívar, 1-90 (2018).

Hansen, J., Sato, M., y Ruedy, R., Perception of climate change, https://doi.org/10.1073/pnas.1205276109, Proceedings of the National Academy of Sciences of the United States of America, 109(37), E2415-E2423 (2012).

Hernández-Díaz, P. M., Polanco, J. A., Escobar-Sierra, M., y Leal, W., Holistic integration of sustaintability at universities: Evidences from Colombia, https://doi.org/10.1016/j.jclepro.2021.127145, Journal of cleaner production, 305, 127-145 (2021).

Hernández-Narváez, D., Vega-Cabrera, A., Zamora-Bornachera, A., y Sierra-Correa, P. C., El cambio climático y los impactos socioeconómicos sobre la zona costera e insular colombiano,

https://doi.org/10.25268/bimc.invemar.2019.48.2.764, Boletín de Investigaciones Marinas y Costeras - INVEMAR, 48 (2), 9-32 (2019)

Hess, D. J., y Maki, A., Climate change belief, sustainability education, and political values: Assessing the need for higher-education curriculum reform, https://doi.org/10.1016/j.jclepro.2019.04.291, Journal of Cleaner Production, 228, 1157-1166 (2019)

Lambrechts, W., y Ceulemans, K., Sustainability Assessment in Higher Education: Evaluating the Use of the Auditing Instrument for Sustainability in Higher Education (AISHE) in Belgium, In Sustainability assessment tools in higher education institutions: mapping trends and good practices around the world, 157-174, Springer International Publishing, Cardiff, United Kingdom (2013).

Leal Filho, W., Ruiz, V., y otros 23 autores., The Role of Higher Education Institutions in Sustainability Initiatives at the Local Level, https://doi.org/10.1016/j.jclepro.2019.06.059, Journal of Cleaner Production, 233, 1004-1015 (2019).

Lemée, C., Navarro, O., y otros 3 autores., Protective behaviors regarding coastal flooding risk in a context of climate change, https://doi.org/10.1016/j.accre.2020.12.001, Advances in Climate Change Research, 11(4), 310-316 (2020).

Luna-Cabrera, G., Narvaez-Romo, A., y Molina-Moreno, A. Percepción de jóvenes rurales frente al ecoturismo en el Centro Ambiental Chimayoy; Municipio de Pasto, Colombia, http://dx.doi.org/10.4067/S0718-07642020000200229, Información tecnológica, 31 (2), 229-238 (2020)

MEN-Ministerio de Educación Nacional. Matriculados en Educación Superior - Colombia 2016, Sistema Nacional de Información de la Educación Superior - SNIES, (2017). 
Moyano, E., Palomo, G., y Moyano, P., Environmental beliefs and ideology in Chilean population. Universum, 30(2), 219236 (2015).

Rangel-Ch, O., La biodiversidad de Colombia: Significado y distribución regional, https://dx.doi.org/10.18257/raccefyn.136, Revista de la Academia Colombiana de Ciencias Exactas, Físicas y Naturales, 39 (151), 176-200 (2015)

Sánchez, F. A., Fundamentos epistémicos de la investigación cualitativa y cuantitativa: Consensos y disensos, https://orcid.org/0000-0002-0144-9892, Revista digital de Investigación en Docencia Universitaria, 13(1), $102-122$ (2019).

Schneiderbauer, S., Fontanella, P, y otros siete autores., Risk perception of climate change and natural hazards in global mountain regions: A critical review., https://doi.org/10.1016/j.scitotenv.2021.146957., Science of the total environment., 784, 146957 (2021)

Viguera, B., Alpízar, F., y otros tres autores., Climate change perceptions and adaptive responses of small-scale coffee farmers in Costa Rica, https://doi.org/10.15517/am.v30i2.32905, Agronomy Mesoamerican, 30(2), 333-351 (2019).

Yáñez, R., Díaz, A. y Pérez, M., Conceptualización y medición de la conducta ciudadana en la carrera en estudiantes universitarios., http://dx.doi.org/10.4067/S0718-50062018000200065, Formación Universitaria, 11 (2), 65-76. (2018)

Yu, H., Wang, B., y otros tres autores., Public perception of climate change in China: results from the questionnaire survey, DOI 10.1007/s11069-013-0711-1, Natural Hazards, 69(1), 459-472 (2013).

Ventura, J., Paiva, J., y otros cinco autores., Urban climate and environmental perception about climate change in Belém - Pará, Brazil, https://doi.org/10.1016/j.uclim.2019.100579, Urban Climate, 31, 100579. (2020) 